\title{
Prognostic models for immunotherapy: emerging factors for an evolving treatment landscape
}

\author{
Howard West \\ Department of Medical Oncology, City of Hope Comprehensive Cancer Center, Duarte, CA, USA \\ Correspondence to: Howard West, MD. Associate Clinical Professor, Department of Medical Oncology, City of Hope Comprehensive Cancer Center, \\ Duarte, CA, USA. Email: hwest@coh.org. \\ Comment on: Prelaj A, Proto C, Lo Russo G, et al. Integrating clinical and biological prognostic biomarkers in patients with advanced NSCLC treated \\ with immunotherapy: the DEMo score system. Transl Lung Cancer Res 2020;9:617-28.
}

Submitted Sep 27, 2020. Accepted for publication Oct 22, 2020.

doi: $10.21037 /$ tlcr-2020-17

View this article at: http://dx.doi.org/10.21037/tlcr-2020-17

The introduction of immune checkpoint inhibitors (ICIs) against PD-1/PD-L1 has transformed not only the management of advanced non-small cell lung cancer (NSCLC) but the broad landscape of oncology practice, and its impact continues to extend across cancer types and indications. In parallel with this development of the field of immunotherapy for cancer treatment, we have struggled to identify prognostic markers to help us understand which patients are most likely to do well overall, as well as predictive markers of which patients are more or less likely to derive the greatest benefit specifically from ICIs. These factors can ideally help us clarify how to prioritize ICIs relative to or combined with conventional chemotherapy regimens or, in molecularly enriched subsets, targeted therapy options. From the beginning, various methods and cutoffs for PD-L1 expression of tumor cells, and in some studies the cells in the microenvironment around the tumor, have been explored for their association of clinical benefit from ICIs $(1,2)$. Tumor PD-L1 expression has emerged as a very imperfect biomarker in lung cancer and other tumor types, without clear utility in predicting whether patients would benefit from ICIs as monotherapy in the second line setting for advanced NSCLC, but serving as a cornerstone of our process of identifying the optimal first line treatment approach, at least for patients whose tumor does not harbor a driver mutation (3). High tumor mutational burden $(\geq 10$ mutations per megabase) has also been more recently received a specific cancer-agnostic approval by the FDA as a potentially useful biomarker (4), though its utility remains controversial (5).
But even limiting our focus to first line treatment of patients with EGFR and ALK wild type advanced NSCLC, our practice today currently entails an ever-widening array of treatment alternatives that require us to select among well studied options that include ICI monotherapy, chemo/ immunotherapy combinations with PD-1/PD-L1 inhibitors and potentially also bevacizumab, as well as immunotherapy combinations with a PD-1/PD-L1 inhibitor and a CTLA-4 inhibitor, potentially with addition of standard chemotherapy. Superimposed on the data that highlight so many choices, both oncologists and patients may have clear preferences about whether to pursue chemotherapy or potentially more challenging combinations when a subset of patients can experience profound and prolonged responses to ICI monotherapy. While this is far more common among patients with tumors demonstrating high PD-L1 expression (6), these extended responses remain elusive even in subsets that reach this threshold.

In this setting, there is a clear appeal to prognostic tools to help us better identify which patients with advanced NSCLC are most likely to do well with ICIs. The DEMo score system that was developed and evaluated by Prelaj and colleagues (7) is a composite of three independent components that each offer some prognostic insights in this realm: the Di Maio $(8,9)$ and EPSILoN $(10)$ scores are based on a collection of clinical variables, along with microRNA signature classifier as a biomarker (11). By aggregating the results of each of these factors that each have an individual score of $1-3$, the DEMo score is reported as a total of $3-9$, creating seven discrete groups that are prognostic for 
both progression-free survival (PFS) and overall survival (OS), though with overlap of the curves, when looking at outcomes of this model applied prospectively to a cohort of 200 patients with advanced NSCLC who were treated with ICI monotherapy, the majority in the second line setting. The median OS of these 7 groups varied from 1.5 months in the least favorable prognostic group to 29.7 months in the most favorable prognostic group. Response rates also followed a similar pattern, ranging from $3 \%$ to $45 \%$ in these 2 groups on the extremes, respectively. The authors also developed models using DEMo results to identify subgroups of patients with high PD-L1 ( $\geq 50 \%$ ) who are far less likely to benefit from ICIs (25\% of that population) as well as a minority (11\%) of patients with low or negative PD-L1 who nevertheless do well with ICI monotherapy.

This work provides a valuable glimpse into next steps of how we can refine our clinical decision-making with a framework like DEMo. Using such a system, we can envision a way to identify with far more confidence which patients with tumors demonstrating high PD-L1 expression can be spared the adverse effects of chemotherapy or immunotherapy combinations when pembrolizumab or atezolizumab monotherapy has a strong probability of providing sustained clinical benefit. Conversely, with many patients eager to avoid chemotherapy and potentially also with relative contraindications to conventional chemotherapy, there is great appeal to having the ability to identify a subset of patients for whom pembrolizumab may be a stronger alternative despite its appropriately nonpreferred status in the broader population of patients with low PD-L1 expression (3).

We must also acknowledge the limitations and shortcomings of this work. The authors acknowledge that this analysis does not include a control group of patients treated by our best currently available tools. The clinical factors of performance status, the overall extent and distribution of disease and tempo of progression of cancerrelated symptoms, and potentially other factors that help comprise the DEMo score are already part of the informal, subjective process clinicians employ in their clinical decision making in routine practice. In addition, DEMo testing includes plasma RNA testing, incurring the turnaround time and cost of this platform. Greater complexity has the potential to refine the prognostic and predictive utility of potential models, that have been developed and include data on as many as 36 multiomics data points-including immune, neoantigen, and checkpoint target variables to accurately predict response to PD-1/PD-L1 inhibitors (12).
In contrast, the Lung Immune Prognostic Index (LIPI), incorporating only the neutrophil/(leukocyte - neutrophil ratio) and measurement of $\mathrm{LDH}$, has been studied and demonstrated to be significantly prognostic for benefit of ICI monotherapy $(13,14)$. We can only evaluate the incremental benefit of DEMo by seeing it used in a randomized trial compared to current best management and strategies like LIPI that require no additional biomarker testing relative to what is readily available in a standard workup.

Another aspect of a prognostic index is the question of how simple and therefore accessible it should be for general use. Aside from plasma microRNA profile testing, the complexity of the DEMo model incorporates a wide range of factors to produce a composite score stratifying patients into seven different groups. Is such a process more cumbersome and complex than would be considered broadly acceptable for routine use outside of the single tertiary care center at which this work was conducted? Notably, only a minority of the patients fall in the two extremes of the best or least favorable prognostic groups, and there is marked overlap in the outcomes for the groups in the gray zone between them. Prelaj and colleagues aggregate some of these groups for specific analyses, but we need to see the proven utility of DEMo relative to current clinical practice or alternate prognostic indices like the LIPI that are far easier to calculate and yield a result that clearly segments patients into just one of three groups corresponding to good, intermediate, or poor prognosis.

There are a few other challenges to this work to consider. The Di Miao scoring system was developed as a prognostic approach for second line treatment $(8,9)$, and this work on the DEMo model includes primarily patients who received ICI monotherapy as second line or later therapy. Management of advanced NSCLC has evolved significantly from that standard, with the vast majority of patients now receiving ICIs either alone or combined with other agents as first line treatment. We cannot presume that we should extrapolate the results of work from second line ICI monotherapy to these revised practice patterns. In addition, this work on the DEMo scoring system does not include consideration of whether a patient's tumor harbors a driver mutation, despite the fact that patients with NSCLC whose tumors harbor a driver mutation are the leading population for whom the question of whether there is any absolute benefit to ICI monotherapy is a highly relevant question.

It is also important to clarify whether the DEMo scoring system is specifically predictive of particular utility of ICI 
monotherapy compared to other competing treatment options or is primarily prognostic of better or worse clinical outcome regardless of the treatment administered. As previously noted, the Di Maio scoring system was developed based on its prognostic role relative to second line chemotherapy $(8,9)$; the LIPI scoring system has also been shown to be prognostic in patients who receive not only immunotherapy but targeted therapy and standard chemotherapy for advanced NSCLC (15). The DEMo system is predictive not only of PFS and OS but also response to ICI monotherapy, but it remains to be determined whether it is distinctly predictive of benefit with this strategy. While a prognostic scoring system that is applicable independent of treatment administered is helpful, the greatest utility is achieved with a predictive model that differentiates a treatment approach that is now likely to be far more or less active than leading alternatives for a specific patient.

Prognostic indices like DEMo have the potential to reveal a subgroup of patients who are expected to have a particularly poor outcome with ICI monotherapy. It remains unclear how to best use this information. First, the response rate in this subgroup with the least favorable DEMo score is not zero, but rather is $3 \%$, and additional patients may experience a modest benefit reflected in stable disease or a minor response. Immunotherapy is sometimes characterized as a "wild card" with the potential for a prolonged benefit, even if relatively unlikely. It is hard to envision many patients forgoing even a small chance for a favorable outcome with immunotherapy, though perhaps identifying a very unfavorable prognosis with ICI monotherapy will direct these patients to combinations that include PD-1/ PD-L1 inhibitors. Importantly, the results available thus far do not offer any insight about whether these patients will have a better outcome with such a combination.

Overall, this work represents an important effort and step forward. By highlighting the potential of prognostic testing to improve on our current limited and crude prognostic factors we rely on today, it suggests a path to identify which patients can most reliably defer chemotherapy and have the highest probability of responding well to ICI monotherapy, while simultaneously indicating a subset of patients who are prone to do poorly despite ICI monotherapy, in whom a combination or perhaps favoring a prioritization of supportive care may be a stronger approach. In the meantime, it will be necessary to demonstrate the feasibility of the DEMo scoring system in a multicenter trial that also translates this prognostic information into better outcomes than either current routine practice today with readily available clinical information or simpler prognostic indices like the LIPI. In addition, it is necessary for the DEMo scoring system to prove its value in a landscape for advanced NSCLC that has overwhelmingly transitioned from second line ICI monotherapy to first line immunotherapy, either alone or combined with conventional chemotherapy, other immunotherapy agents, or both. With so many competing and reasonable options, a clinical tool to help identify the optimal path to avoid both undertreatment and overtreatment is a remarkably promising concept. Future work here can help us determine if we will be able to avail ourselves of such a refinement of our current approach to decision making in advanced NSCLC in the coming years.

\section{Acknowledgments}

Funding: None.

\section{Footnote}

Provenance and Peer Review: This article was commissioned by the editorial office, Translational Lung Cancer Research. The article did not undergo external peer review.

Conflicts of Interest: The author has completed the ICMJE uniform disclosure form (available at http://dx.doi. org/10.21037/tlcr-2020-17). Dr. HW reports personal fees from AstraZeneca, personal fees from Genentech/Roche, personal fees from Merck, outside the submitted work.

Ethical Statement: The author is accountable for all aspects of the work in ensuring that questions related to the accuracy or integrity of any part of the work are appropriately investigated and resolved.

Open Access Statement: This is an Open Access article distributed in accordance with the Creative Commons Attribution-NonCommercial-NoDerivs 4.0 International License (CC BY-NC-ND 4.0), which permits the noncommercial replication and distribution of the article with the strict proviso that no changes or edits are made and the original work is properly cited (including links to both the formal publication through the relevant DOI and the license). See: https://creativecommons.org/licenses/by-nc-nd/4.0/.

\section{References}

1. Davis AA, Patel VG. The role of PD-L1 expression as a 
predictive biomarker: an analysis of all US Food and Drug Administration (FDA) approvals of immune checkpoint inhibitors. J Immunother Cancer 2019;7:278.

2. Schoenfeld AJ, Rizvi H, Bandlamudi C, et al. Clinical and molecular correlates of PD-L1 expression in patients with lung adenocarcinomas. Ann Oncol 2020;31:599-608.

3. National Comprehensive Cancer Network (NCCN) Clinical Practice Guidelines in Oncology (NCCN Guidelines): Non-Small Cell Lung Cancer (version 8.2020) [Internet] 2020 Sept [cited 2020 Sept 25]. Available online: https://bit.ly/NCCNNSCLC

4. FDA approves pembrolizumab for adults and children with TMB-H solid tumors. [Internet] 2020 Jun [cited 2020 Sept 25]. Available online: https://www.fda.gov/drugs/drugapprovals-and-databases/fda-approves-pembrolizumabadults-and-children-tmb-h-solid-tumors

5. Addeo A, Banna GL, Weiss GJ. Tumor Mutation BurdenFrom Hopes to Doubts. JAMA Oncol 2019;5:934-5.

6. Reck M, Rodríguez-Abreu D, Robinson AG, et al. Updated Analysis of KEYNOTE-024: Pembrolizumab Versus Platinum-Based Chemotherapy for Advanced NonSmall-Cell Lung Cancer With PD-L1 Tumor Proportion Score of 50\% or Greater. J Clin Oncol 2019;37:537-46.

7. Prelaj A, Proto C, Lo Russo G, et al. Integrating clinical and biological prognostic biomarkers in patients with advanced NSCLC treated with immunotherapy: the DEMo score system. Transl Lung Cancer Res 2020;9:617-28.

8. Di Maio M, Lama N, Morabito A, et al. Clinical assessment of patients with advanced non-small-cell lung cancer eligible for second-line chemotherapy: a prognostic score from individual data of nine randomised trials. Eur J

Cite this article as: West H. Prognostic models for immunotherapy: emerging factors for an evolving treatment landscape. Transl Lung Cancer Res 2021;10(1):9-12. doi: 10.21037/tlcr-2020-17
Cancer 2010;46:735-43.

9. Di Maio M, Krzakowski M, Fougeray R, et al. Prognostic score for second-line chemotherapy of advanced nonsmall-cell lung cancer: external validation in a phase III trial comparing vinflunine with docetaxel. Lung Cancer 2012;77:116-20.

10. Prelaj A, Ferrara R, Rebuzzi SE, et al. EPSILoN: A Prognostic Score for Immunotherapy in Advanced NonSmall-Cell Lung Cancer: A Validation Cohort. Cancers (Basel) 2019;11:1954.

11. Boeri M, Milione M, Proto C, et al. Circulating miRNAs and PD-L1 Tumor Expression Are Associated with Survival in Advanced NSCLC Patients Treated with Immunotherapy: a Prospective Study. Clin Cancer Res 2019;25:2166-73.

12. Lee JS, Ruppin E. Multiomics Prediction of Response Rates to Therapies to Inhibit Programmed Cell Death 1 and Programmed Cell Death 1 Ligand 1. JAMA Oncol 2019;5:1614-8.

13. Mezquita L, Auclin E, Ferrara R, et al. Association of the Lung Immune Prognostic Index with immune checkpoint inhibitor outcomes in patients with advanced non-small cell lung cancer. JAMA Oncol 2018;4:351-7.

14. Banna GL, Signorelli D, Metro G, et al. Neutrophil-tolymphocyte ratio in combination with $\mathrm{PD}-\mathrm{L} 1$ or lactate dehydrogenase as biomarkers for high PD-L1 non-small cell lung cancer treated with first-line pembrolizumab. Transl Lung Cancer Res 2020;9:1533-42.

15. Kazandjian D, Gong Y, Keegan P, et al. Prognostic value of the Lung Immune Prognostic Index for patients treated for metastatic non-small cell lung cancer. JAMA Oncol 2019;5:1481-5. 